\title{
Paralemmin-3 contributes to lipopolysaccharide-induced inflammatory response and is involved in lipopolysaccharide- Toll-like receptor-4 signaling in alveolar macrophages
}

\author{
XU-XIN CHEN ${ }^{1 *}$, LU TANG $^{2 *}$, YU-MEI FU ${ }^{3 *}$, YI WANG $^{4}$, ZHI-HAI HAN ${ }^{1}$ and JI-GUANG MENG ${ }^{1}$ \\ ${ }^{1}$ Department of Respiratory Medicine, Navy General Hospital of the PLA, Beijing 100037; ${ }^{2}$ Department of Neurology, \\ The First Hospital of Changsha, Changsha, Hunan 430100; ${ }^{3}$ Department of Emergency, The First Affiliated Hospital \\ of Henan University of Science and Technology, Luoyang, Henan 471003; ${ }^{4}$ Department of Respiratory Medicine, \\ The Sixth People's Hospital of Jinan City Affiliated to Jining Medical College, Jinan, Shandong 250200, P.R. China
}

Received March 14, 2017; Accepted September 19, 2017

DOI: $10.3892 / \mathrm{ijmm} .2017 .3161$

\begin{abstract}
Alveolar macrophages (AMs) are the first line of defense against foreign stimulation in alveoli, and they participate in inflammatory responses during acute lung injury (ALI). Previous studies indicated that paralemmin-3 (PALM3) expression is induced by lipopolysaccharides (LPS) and may be involved in LPS-Toll-like receptor 4 (TLR4) signaling in alveolar epithelial cells. The aim of the present study was to investigate the effect of PALM3 on LPS-induced inflammation and its underlying mechanisms in rat AMs. For this purpose, the authors detected the expression of PALM3 in AMs by reverse transcription-quantitative polymerase chain reaction (RT-qPCR) and western blotting following LPS stimulation. Following this, a recombinant adenovirus expressing short
\end{abstract}

Correspondence to: Professor Zhihai Han or Professor Jiguang Meng, Department of Respiratory Medicine, Navy General Hospital of the PLA, 6 Fucheng Road, Beijing 100037, P.R. China

E-mail: zhihaihandoctor@163.com

E-mail:mjg2016@tom.com

*Contributed equally

Abbreviations: ALI, acute lung injury; AM, alveolar macrophage; AP-1, activator protein-1; ARDS, acute respiratory distress syndrome; IFN, interferon; IL, interleukin; IRAK, interleukin 1 receptor associated kinase; IRF-3, IFN regulatory factor 3; LBP, LPS-binding protein; LPS, lipopolysaccharide; MAL, MyD88-adaptor-like; TLR, Toll-like receptor; MAPK, mitogen-activated protein kinase; MyD88, myeloid differentiation factor 88 ; NF- $\mathrm{BB}$, nuclear transcription factor $\kappa \mathrm{B}$; PALM3, parelemmin-3; SIGIRR, single immunoglobulin IL-1 receptor-related molecule; TIACM, Toll-IL-1 receptor containing adapter molecule; TNF, tumor necrosis factor; TRAF, tumor necrosis factor receptor associated factor; TRIF, TIR domain-containing adaptor inducing IFN- $\beta$

Key words: paralemmin-3, lipopolysaccharide, Toll-like receptor, acute lung injury, acute respiratory distress syndrome, alveolar macrophages, nuclear factor- $\kappa \mathrm{B}$, interferon regulatory factor 3 , co-immunoprecipitation hairpin RNA (shRNA) for PALM3 was constructed, as well as a recombinant adenovirus carrying the rat $P A L M 3$ gene to modulate the expression of PALM3 in rat AMs. At $48 \mathrm{~h}$ after transfection, the PALM3 expression in AMs was detected by RT-qPCR and western blotting. The levels of several cytokines and the activity of nuclear factor- $\kappa \mathrm{B}$ and interferon regulatory factor 3 in AMs were measured after LPS stimulation. The localization of PALM3 and LPS-TLR4 signaling adaptor molecules in AMs was analyzed by confocal microscopy, and the physical interactions of PALM3 with these adaptors were assessed by co-immunoprecipitation assays. LPS induced PALM3 expression in AMs and that PALM3 expression promoted the LPS-induced inflammatory response, while PALM3 downregulation suppressed the LPS-induced inflammatory response in AMs. In addition, the results demonstrated that PALM3 could interact with TLR4, myeloid differentiation factor 88, interleukin (IL)-1 receptor associated kinase-1, tumor necrosis factor receptor associated factor-6, and Toll-IL-1 receptor containing adapter molecule-2 in AMs after LPS stimulation. These results suggested that PALM3 contributes to the LPS-induced inflammatory response and participates in LPS-TLR4 signaling in AMs. These data may provide the basis for the development of novel targeted therapeutic strategies of treating ALI.

\section{Introduction}

Acute lung injury (ALI) and its severe form acute respiratory distress syndrome (ARDS) are devastating clinical syndromes that frequently lead to respiratory failure characterized by neutrophil accumulation, diffuse endothelium and epithelial damage, air-blood barrier disruption, and the subsequent infiltration of peripheral inflammatory cells into lung tissues (1). Currently, there is no effective pharmacological therapy for ALI/ARDS, and the morbidity and mortality rates of ARDS remain as high as $30-40 \%$ (2). The pathophysiological mechanism of ALI/ARDS is believed to be associated with an uncontrolled and excessive inflammatory response in lungs (3). An unchecked inflammatory reaction and the presence of excessive inflammatory mediators can lead to inappropriate immunity-related tissue damage, including ALI. 
Alveolar macrophages (AMs) are the most abundant cells in the alveoli, distal airspaces and conducting airways (4). They form the first line of defense against airborne particles and microbes. AMs account for $90 \%$ of the cells in bronchoalveolar lavage fluid; as the main immune cells, they recognize pathogen-associated molecular patterns and trigger innate immunity and host defenses $(4,5)$. Previous studies have demonstrated that macrophages contribute to the modulation of inflammatory responses during ALI/ARDS as well as to the resolution of inflammation and tissue repair in lungs $(5,6)$. With increased foreign antigen stimulation, the macrophages become activated, which further leads to inflammatory cytokine secretion, neutrophil recruitment and T-effector cell interaction (7). Lipopolysaccharides (LPS), an important component of the outer wall of Gram-negative bacteria, are highly proinflammatory molecules that can activate AMs, stimulating their production of inflammatory mediators $(8,9)$. LPS is recognized by LPS-binding protein (LBP) and CD14, which then bind to Toll-like receptor 4 (TLR4). Following this, several adaptor proteins are recruited to transduce multiple key downstream intracellular signaling pathways, such as the mitogen-activated protein kinase (MAPK), IFN regulatory factor 3 (IRF-3), and nuclear factor (NF) $-\kappa \mathrm{B}$ cascades. Activation of these signaling pathways eventually induces the production of various cytokines, including tumor necrosis factor (TNF)- $\alpha$, interleukin (IL)-1 $\beta$, interferon (IFN)- $\alpha$ and IFN- $\beta(10,11)$. Despite their beneficial effects during infection, excessive production of inflammatory mediators may cause edema, cell necrosis, tissue damage and other pathological changes (12).

Paralemmin-3 (PALM3) was first described in Xenopus laevis as Xlgv7/Xlcaax-1 by Cornish et al (13) and belongs to the paralemmins (PALMs) protein family. The PALM protein family includes PALM1, PALM2, PALM3 and palmdelphin (PALMD) (14), and these proteins are highly expressed in the brain, kidney, adrenal gland and mammary gland, as well as in breast cancers (15-17). Previous studies have implicated PALM1 as having a role in cell shape control, plasma membrane dynamics, cell motility, cancer cell invasiveness and metastatic potential, cell migration and maturation modulation, and tumor lymphangiogenesis $(15,16,18)$. However, PALM3 is speculated to act as an adaptor to link intrinsic membrane proteins to each other, to the cytoskeleton, or to motor proteins $(14,19)$. Previous work of the authors revealed that LPS can upregulate PALM3 expression in alveolar epithelial cells (19). In addition, the study revealed that PALM3 expression is induced by LPS and may be involved in the LPS-TLR4 signaling in alveolar epithelial cells (A549 cells) (19). The downregulation of PALM3 is able to ameliorate LPS-induced ALI in mice and reduce the LPS-induced inflammatory response in alveolar epithelial cells $(19,20)$. However, whether or not PALM3 participates in LPS-TLR4 signaling in AMs is still unclear. Additionally, the manner in which PALM3 participates in TLR4 signal transduction also warrants further investigation. Therefore, the present study was conducted to investigate the role of PALM3 in LPS-TLR4 signal transduction using a rat NR8383 macrophage model, which can be stimulated with LPS to mimic an inflammatory state. For this purpose, the authors aimed to modulate the expression of PALM3 in AMs by using recombinant adenoviral vectors. To assess the effects of PALM3 expression, the corresponding cytokine levels were observed, as well as the activity of NF- $\kappa$ B and IRF-3 in AMs. They detected the interaction of PALM3 with adaptor molecules in LPS-TLR4 signaling by co-immunoprecipitation and confocal microscopy.

\section{Materials and methods}

Cell culture. Cells (293) were obtained from Microbix Biosystems, Inc. (Toronto, Canada) and were grown in Dulbecco's modified Eagle's medium (Gibco; Thermo Fisher Scientific, Inc., Waltham, MA, USA), supplemented with $10 \%$ fetal bovine serum (Gibco; Thermo Fisher Scientific, Inc.) at $37^{\circ} \mathrm{C}$ in a humidified chamber with $5 \% \mathrm{CO}_{2}$. The rat $\mathrm{AM}$ cell line NR8383 (American Type Culture Collection, Manassas, VA, USA) was cultured in Ham's F12 medium (Gibco; Thermo Fisher Scientific, Inc.), supplemented with $10 \%$ fetal bovine serum at $37^{\circ} \mathrm{C}$ in a humidified atmosphere with $5 \% \mathrm{CO}_{2}$. Cells in the exponential growth phase were used in the experiments described in the following section.

Generation of adenoviral vectors. An adenovirus was generated that contained a single open reading frame encoding rat PALM3 (rPALM3). First, cDNA coding for rPALM3 was synthesized by GeneChem Co. Ltd. (Shanghai, China) and ligated into the adenoviral shuttle plasmid pDC316 (Microbix Biosystems, Inc.) to generate the plasmid pDC316-rPALM3. Recombinant viruses were then generated using 293 packaging cells co-transfected with pDC316-rPALM3 and a plasmid containing cDNA for adenoviral proteins (pBHGlox; Microbix Biosystems, Inc.) via the $\mathrm{AdMax}^{\mathrm{TM}}$ system (Microbix Biosystems, Inc.). Plaques were isolated after $\sim 14$ days and expanded in 293 cells. Viral stocks were purified using $\mathrm{CsCl}$ gradients. The acquired overexpression adenoviral vector was named Ad.rPALM3. Meanwhile, a recombinant adenoviral vector expressing short hairpin (sh)RNA for rat PALM3 was constructed using a similar method and named Ad.shRNA. The sequence 5'-AGATCTTGATGGAGGGTTT-3' was chosen as the targeted sequence. The primers for the targeted sequence were: 5'-CCGGGGAGATCTTGATGGAGGGT TTCTCGAGAAACCCTCCATCAAGATCTCCTTTTTG-3' (sense) and 5'-AATTCAAAAAGGAGATCTTGATGGAGG GTTTCTCGAGAAACCCTCCATCAAGATCTCC-3' (antisense) (GeneChem Co., Ltd.). An adenoviral vector, free of any transgenes, was used as a control vector and named Ad.V (GeneChem Co., Ltd.). The titers of the recombinant adenoviruses were determined by means of the $50 \%$ tissue culture infectious dose. The titers of Ad.rPALM3, Ad.shRNA and Ad.V were $5 \times 10^{9}$ plaque-forming units $(\mathrm{PFU}) / \mathrm{ml}, 3 \times 10^{10} \mathrm{PFU} / \mathrm{ml}$, and $6 \times 10^{9} \mathrm{PFU} / \mathrm{ml}$, respectively.

Experimental design. Rat AMs were routinely grown in Ham's F12 medium supplemented with $10 \%$ fetal bovine serum and seeded in 6-well plates at a density of $1 \times 10^{5}$ cells/well. After the cell growth reached 50-60\% confluence, rat AMs were treated with $0.5 \mu \mathrm{g} / \mathrm{ml}$ LPS (Escherichia coli 0111: B4; Sigma-Aldrich; Merck KGaA, Darmstadt, Germany) as described previously to simulate ALI in an in vitro model $(4,5)$. The total RNA and protein were isolated before the treatment with LPS and at 3,6, 12, 24 and $48 \mathrm{~h}$ following the addition of LPS for the analysis of PALM3 expression. 
For some experiments, rat AMs were seeded in appropriate plates and left untreated (normal) or were transfected with the adenoviruses Ad.rPALM3, Ad.shRNA, or Ad.V when the cell growth reached $50-60 \%$ confluence. At $48 \mathrm{~h}$ post-transfection, the total RNA and protein were isolated from some of the cells for detecting the PALM3 expression in cells transfected with the recombinant adenoviruses. Also, at $48 \mathrm{~h}$ post-transfection, $0.5 \mu \mathrm{g} / \mathrm{ml}$ LPS was added into the cell culture medium of some cells (normal + LPS, Ad.rPALM3 + LPS, Ad.shRNA + LPS and Ad.V + LPS). The cell culture supernatant was collected both before the LPS treatment and at $24 \mathrm{~h}$ following LPS stimulation for the detection of cytokine levels, and the nuclear protein was isolated for determining the activity of NF- $\kappa \mathrm{B}$ and IRF-3.

For other experiments, rat AMs were seeded in appropriate plates and, after the cells were treated with LPS $(0.5 \mu \mathrm{g} / \mathrm{ml})$ or phosphate-buffered saline (PBS) for $24 \mathrm{~h}$, the total protein was extracted for subsequent co-immunoprecipitation, and cell monolayers were fixed with $4 \%$ paraformaldehyde for immunofluorescence analysis.

RNA isolation and reverse transcription-quantitative polymerase chain reaction ( $R T-q P C R)$. Following the treatment, cells were harvested, and total cellular RNA was isolated using TRIzol (Invitrogen; Thermo Fisher Scientific, Inc.) according to the manufacturer's instructions. Reverse transcription (RT) of the lung samples was performed using the Takara PrimeScript RT Reagent kit (Takara Biotechnology Co., Ltd., Dalian, China). Then, RT-qPCR was performed using the iTaq Universal SYBR Green Supermix (Takara Biotechnology Co., Ltd.) according to the manufacturer's instructions. Rat $\beta$-actin was selected as an internal standard. The primers used for rat PALM3 were 5'-GAGGCAGGG ATCTTGATGTC-3' (sense) and 5'-GCCCAACACCCTCA AGACTA-3' (antisense). The primers used for rat $\beta$-actin were 5'-GGAGATTACTGCCCTGGCTCCTA-3' (sense) and 5'-GACTCATCGTACTCCTGCTTGCTG-3' (antisense). The PCR conditions were as follows: $95^{\circ} \mathrm{C}$ for $30 \mathrm{sec}$, then 35 cycles of $95^{\circ} \mathrm{C}$ for $5 \mathrm{sec}, 60^{\circ} \mathrm{C}$ for $30 \mathrm{sec}$. All reactions were performed in triplicate, and reports were generated by Rotor-Gene Real-time Analysis Software 6.0 (Qiagen GmbH, Hilden, Germany). The relative expression of each target gene (PALM3 and $\beta$-actin) was calculated by the $2^{-\Delta \Delta C \mathrm{q}}$ method (21).

Protein extraction and western blotting. Following appropriate treatment, rat AMs were lysed in lysis buffer $[30 \mathrm{mM}$ Tris- $\mathrm{HCl}$ (pH 8.0), $200 \mathrm{mM} \mathrm{NaCl}, 1 \%$ NP-40, $1 \mathrm{mM}$ phenylmethylsulfonyl fluoride and protease inhibitor cocktail (Sigma-Aldrich; Merck KGaA)]. The protein concentrations were measured by the bicinchoninic acid (BCA) assay method (Sigma-Aldrich; Merck KGaA). Equal amounts of protein (30 $\mu \mathrm{g})$ were separated using SDS-PAGE and transferred onto a polyvinylidene difluoride membrane. The membranes were then blocked and incubated overnight at $4^{\circ} \mathrm{C}$ with goat polyclonal anti-rPALM3 antibody (cat. no. sc-248213; polyclonal goat anti-rat; Santa Cruz Biotechnology, Inc., Dallas, TX, USA) at a 1:500 dilution in TBST (TBS with $0.1 \%$ Tween-20). After extensive washing with TBST, the membranes were incubated with horseradish peroxidase-labeled rabbit anti-goat secondary antibody at a 1:2,500 dilution in TBST (cat. no. ZB-2306; Beijing Zhongshan
Golden Bridge Biotechnology Co., Ltd., Beijing, China) at room temperature for $120 \mathrm{~min}$. The signals were detected by enhanced chemiluminescence following the manufacturer's instructions (Beyotime Institute of Biotechnology, Haimen, China). The images were quantified by Quantity One (version, 4.62; Bio-Rad Laboratories, Inc., Hercules, CA, USA) software.

ELISA for the detection of cytokine levels. The expression levels of TNF- $\alpha$, IL-1 $\beta$, IL-10, IFN- $\alpha$, IFN- $\beta$ and macrophage migration inhibitory factor (MIF) were measured by performing ELISAs. The NR8383 AMs were incubated in 24-well plates and transfected with Ad.rPALM3, Ad.shRNA or Ad.V. At $48 \mathrm{~h}$ following transfection, the AMs were treated with LPS $(0.5 \mu \mathrm{g} / \mathrm{ml})$. The cell culture supernatant was collected both before the treatment with LPS and, at $24 \mathrm{~h}$ after the addition of LPS, these samples were centrifuged at $1,000 \mathrm{xg}$ for $15 \mathrm{~min}$. The levels of TNF- $\alpha$ (cat. no. RTA00), IL-1 $\beta$ (cat. no. RLB00) and IL-10 (cat. no. R1000) (R\&D System, Inc., Minneapolis, MN, USA) and of IFN- $\alpha$ (cat. no. CSB-E08637r), IFN- $\beta$ (cat. no. CSB-E04845r) and MIF (cat. no. CSB-E07293r) (Cusabio Biotech Co., Ltd., Wuhan, China) in the supernatants were determined by using the corresponding ELISA kit, according to the manufacturer's instructions.

ELISA for the detection of NF- $\kappa B$ activity. The NR8383 AMs were incubated in 6-well plates and transfected with Ad.rPALM3, Ad.shRNA or Ad.V. At 48 h after transfection, the AMs were treated with LPS $(0.5 \mu \mathrm{g} / \mathrm{ml})$ for $24 \mathrm{~h}$. The nuclear extracts of AMs were then prepared by using the Nuclear Extract kit (Active Motif, Carlsbad, CA, USA) according to the manufacturer's protocol. The protein concentration of the nuclear extracts was quantified by the BCA method (Sigma-Aldrich; Merck KGaA). The NF- $\kappa$ B p65 DNA binding activity in the isolated nuclear extracts was assessed by performing ELISAs using the TransAM ${ }^{\mathrm{TM}} \mathrm{NF}-\kappa \mathrm{B}$ Transcription Factor Assay kit according to the manufacturer's protocol (Active Motif).

Western blot analysis of phospho-IRF3. After the cells were treated as described above, the nuclear extracts of AMs were prepared as described above, and the procedure of western blot analysis was performed as described above. The nuclear extracts were analyzed by immunoblotting with rabbit polyclonal phosphor-specific anti-IRF-3 (1:500 in TBST; cat. no. ab138449; Abcam, Cambridge, UK) antibody. Rabbit polyclonal Histone H3 (cat. no. sc8654; 1:1,000 in TBST; Santa Cruz Biotechnology, Inc.) was used as a lysate control.

Confocal immunofluorescence imaging. Rat AMs were plated on poly-L-lysine-coated glass cover slides and cultured until they reached $50-60 \%$ confluence, after which these cells were treated with LPS $(0.5 \mu \mathrm{g} / \mathrm{ml})$ or PBS for $24 \mathrm{~h}$. Following LPS stimulation, the cell monolayers were fixed with $4 \%$ paraformaldehyde for $20 \mathrm{~min}$, permeabilized with $0.1 \%$ Triton $\mathrm{X}-100$ for $30 \mathrm{~min}$, and blocked with $5 \%$ bovine serum albumin (BSA) for $1 \mathrm{~h}$ at room temperature. Slides were incubated overnight at $4^{\circ} \mathrm{C}$ with a primary rat-specific anti-rPALM3 antibody $(1: 100$ in PBS with $1 \%$ BSA; cat. no. sc-248213; polyclonal goat anti-rat; Santa Cruz Biotechnology, Inc.), anti-TLR4 antibody (1:50 in PBS with 1\% BSA; cat. no. sc-293072; monoclonal 
A

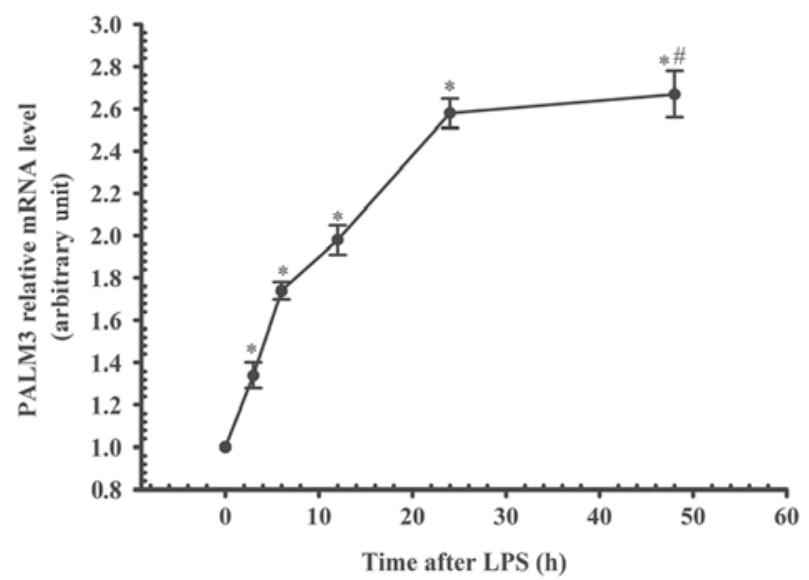

B
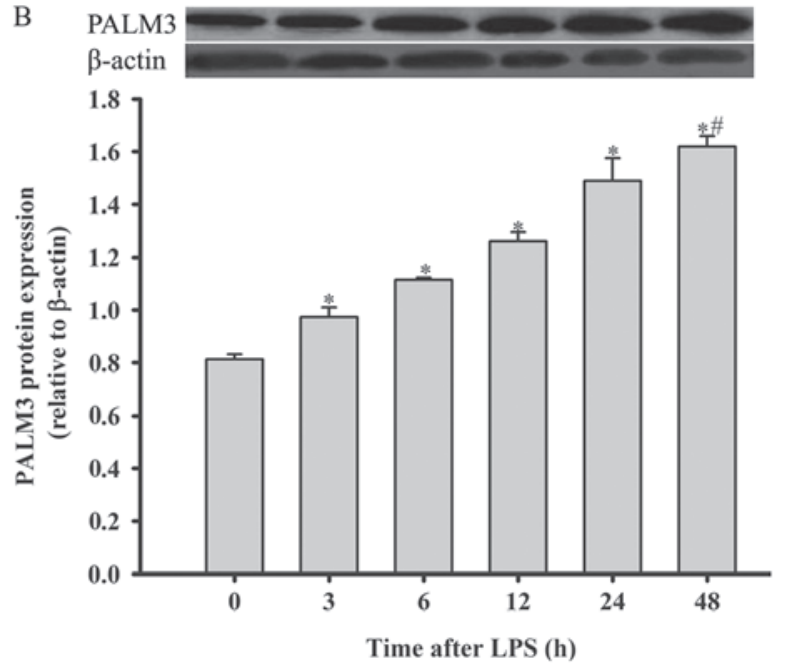

Figure 1. Gene and protein expression of PALM3 in rat AMs following LPS stimulation. The (A) mRNA levels and (B) protein levels of PALM3 in rat AMs at $0,3,6,12,24$ and $48 \mathrm{~h}$ following LPS stimulation were determined by reverse transcription-quantitative polymerase chain reaction and western blotting, respectively. The upper panels of (B) show representative blots, and the lower panels of (B) represent densitometry analyses of the bands. Data are expressed as the mean \pm standard error of the mean of three independent experiments. ${ }^{*} \mathrm{P}<0.05 \mathrm{vs} .0 \mathrm{~h}$ time point; ${ }^{\#} \mathrm{P}>0.05$ for $24 \mathrm{~h}$ time point vs. $48 \mathrm{~h}$ time point. AMs, alveolar macrophages; LPS, lipopolysaccharides.

mouse anti-rat; Santa Cruz Biotechnology, Inc.), anti-myeloid differentiation factor 88 (MyD88; HFL-296) antibody (1:50 in PBS with 1\% BSA; cat. no. sc-11356; monoclonal mouse antirat; Santa Cruz Biotechnology, Inc.), anti-interleukin 1 receptor associated kinase (IRAK)-1 antibody (1:50 in PBS with 1\% BSA; cat. no. NBP1-77068; polyclonal rabbit anti-rat; Novus Biologicals, LLC, Littleton, CO, USA), anti-tumor necrosis factor receptor associated factor (TRAF)-6 antibody (1:100 in PBS with 1\% BSA; cat. no. NBP1-33357; polyclonal rabbit antirat; Novus Biologicals, LLC), or anti-TICAM-2 (E-2) antibody (1:50 in PBS with 1\% BSA; cat.no.sc-376076; monoclonal mouse anti-rat; Santa Cruz Biotechnology, Inc.). Following incubation, the slides were washed three times with PBS. The slides were then incubated at room temperature with a Cy3-conjugated donkey anti-goat secondary antibody at a 1:1,000 dilution (cat. no. A0502; Beyotime Institute of Biotechnology) for 90 min followed by three washes with PBS. The slides were finally incubated with a fluorescein isothiocyanate (FITC)conjugated goat anti-mouse secondary antibody at a 1:1,000 dilution (cat. no. A0568; Beyotime Institute of Biotechnology) or a FITC-conjugated goat anti-rabbit secondary antibody at a 1:1,000 dilution (cat. no. A0562; Beyotime Institute of Biotechnology) for $30 \mathrm{~min}$. The slides were subsequently counterstained for 5 min with 4',6-diamidino-2-phenylindole (DAPI), and samples were observed using a confocal Leica TCS SP5 (Leica Microsystems GmbH, Wetzlar, Germany).

Co-immunoprecipitation. To examine protein-protein interactions, co-immunoprecipitation assays were performed. The rat AMs were treated with LPS $(0.5 \mu \mathrm{g} / \mathrm{ml})$ or PBS for $24 \mathrm{~h}$. Then, the cells were washed with PBS and scraped from the plate in the lysis buffer described above. Aliquots of the resulting cell lysates (containing $500 \mu \mathrm{g}$ protein) were incubated with a primary rat-specific anti-TLR4 (25) antibody (cat. no. sc-293072; 1:100 in TBST; Santa Cruz Biotechnology, Inc.), anti-MyD88 antibody (D80F5; 1:50 in TBST; cat. no. 4283; monoclonal rabbit anti-rat; Cell Signaling Technology, Inc., Danvers, MA, USA), anti-
IRAK-1 antibody (cat. no. NBP1-77068; 1:50 in TBST; Novus Biologicals, LLC), anti-TRAF-6 antibody (cat. no. NBP133357; 1:50 in TBST; Novus Biologicals, LLC), anti-TICAM-2 (E-2) antibody (cat. no. sc-376076; 1:50 in TBST; Santa Cruz Biotechnology, Inc.), or normal rat IgG (cat. no. sc-2026; 1:50 in TBST; Santa Cruz Biotechnology, Inc.) at $4^{\circ} \mathrm{C}$ for $2 \mathrm{~h}$ and then with $20 \mu \mathrm{l}$ protein $\mathrm{A} / \mathrm{G}$-agarose (Beyotime Institute of Biotechnology) at $4^{\circ} \mathrm{C}$ with rocking overnight. The pellets obtained after centrifugation at $14,000 \mathrm{x}$ g for $5 \mathrm{sec}$ were washed five times with washing buffer [50 mM Tris ( $\mathrm{pH} 7.5)$, $7 \mathrm{mM} \mathrm{MgCl} 2,2 \mathrm{mM}$ EDTA and $1 \mathrm{mM}$ PMSF (phenylmethylsulfonyl fluoride)]. The pellets were resolved by $1 \mathrm{X}$ SDS-PAGE loading buffer and boiled for $10 \mathrm{~min}$. Following centrifugation, the supernatants were obtained as immunoprecipitates for western blotting analysis by using anti-PALM3 antibody, antiTLR4 antibody, anti-MyD88 antibody, anti-IRAK-1 antibody, anti-TRAF-6 antibody or anti-TICAM-2 antibody.

Statistical analysis. All data are presented as means \pm standard error of the mean. Comparison of means was performed by a one-way analysis of variance, and the Student-NewmanKeuls test was used to analyze comparisons between multiple groups. $\mathrm{P}<0.05$ was considered to indicate a statistically significant difference.

\section{Results}

Expression of PALM3 after LPS stimulation in rat AMs. The authors detected PALM3 gene and protein expression in rat AMs by using RT-qPCR and western blot analysis at 0,3 , 6, 12, 24 and $48 \mathrm{~h}$ following LPS stimulation. The PALM3 gene and protein expression in rat AMs were both upregulated following the administration of LPS in a time-dependent manner ( $\mathrm{P}<0.05$ vs. their respective $0 \mathrm{~h}$ time points; Fig. 1). However, there was no significant difference in the PALM3 expression levels between the 24 and $48 \mathrm{~h}$ time points $(\mathrm{P}>0.05$ for the $24 \mathrm{~h}$ time point vs. $48 \mathrm{~h}$ time point; Fig. 1). 
A

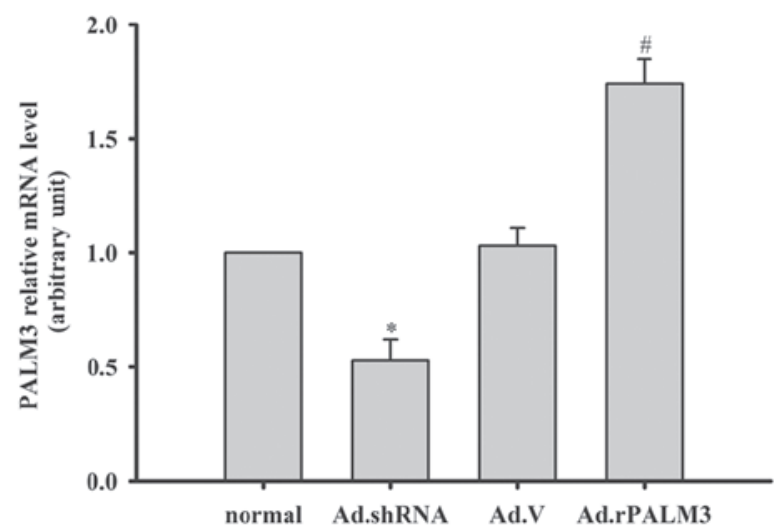

B
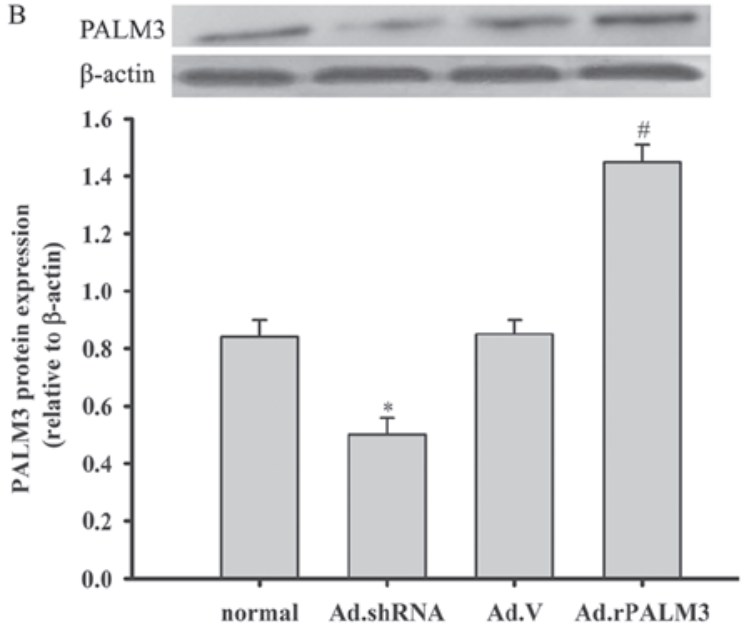

Figure 2. Expression of PALM3 after adenovirus transfection in rat AMs. The (A) mRNA levels and (B) protein levels of PALM3 in untransfected (normal) or adenovirus-transfected rat AMs at $48 \mathrm{~h}$ following transfection with Ad.shRNA, Ad.V, or Ad.rPALM3 were determined by reverse transcription-quantitative polymerase chain reaction and western blotting, respectively. The upper panels (B) show representative blots, and the lower panels (B) represent densitometry analyses of the bands. Data are expressed as the mean \pm standard error of the mean of three independent experiments. " $\mathrm{P}<0.05$ vs. Ad.V and normal groups; ${ }^{\text {"P}} \mathrm{P}<0.05$ vs. Ad.V and normal groups. AMs, alveolar macrophages; shRNA, short hairpin RNA; Ad, adenovirus; normal, untransfected cells; Ad.shRNA, Ad.shRNA-transfected cells; Ad.V, Ad.V-transfected cells; Ad.rPALM3, Ad.rPALM3-transfected cells.

Expression of PALM3 after adenovirus transfection in rat $A M s$. At $48 \mathrm{~h}$ following adenovirus transfection, the total RNA and protein of rat AMs were isolated for an analysis of the PALM3 expression. Compared with the normal (untransfected) and Ad.V (Ad.V-transfected as a negative control) groups, the PALM3 mRNA and protein levels in rat AMs in the Ad.rPALM3 (Ad.rPALM3-tranfected) group were significantly enhanced $(\mathrm{P}<0.05$ vs. normal and Ad.V groups; Fig. $2 \mathrm{~A}$ and B). However, in the Ad.shRNA (Ad.shRNA-transfected) group, the PALM3 mRNA and protein expression were significantly inhibited ( $\mathrm{P}<0.05$ vs. normal and Ad.V groups; Fig. 2A and B).

Effect of PALM3 on the production of cytokines in $L P S$-stimulated rat AMs. The influence of PALM3 on the levels of TNF- $\alpha$, IL-1 $\beta$, IL-10, IFN- $\alpha$, IFN- $\beta$ and MIF was measured by ELISAs in LPS-stimulated NR8383 cells. The ELISA results show that the levels of TNF- $\alpha$, IL-1 $\beta$, IL-10, IFN- $\alpha$, IFN- $\beta$ and MIF were very low in the culture supernatant of LPS-unstimulated cells (Fig. 3). The administration of Ad.V, Ad.shRNA or Ad.rPALM3 did not influence the level of cytokine (TNF- $\alpha$, IL-1 $\beta$, IL-10, IFN- $\alpha$, IFN- $\beta$ and MIF) release before LPS stimulation ( $\mathrm{P}>0.05$ vs. normal cells; Fig. 3). However, in response to LPS, there was a marked increase in the levels of TNF- $\alpha$, IL- $1 \beta$, IL-10, IFN- $\alpha$, IFN- $\beta$, and MIF in all LPS-stimulated cells $(\mathrm{P}<0.05$ vs. unstimulated cells). Ad.rPALM3 pretreatment increased the amount of proinflammatory cytokines (TNF- $\alpha$, IL- $1 \beta$, IFN- $\alpha$, IFN- $\beta$ and MIF) but decreased the amount of an anti-inflammatory cytokine (IL-10) released from rat AMs after LPS stimulation $(\mathrm{P}<0.05$ vs. Ad.V + LPS, normal + LPS, and Ad.shRNA + LPS groups; Fig. 3). Downregulation of PALM3 through Ad.shRNA pretreatment inhibited LPS-induced expression of these proinflammatory cytokines but promoted LPS-induced expression of an anti-inflammatory cytokine (IL-10) $(\mathrm{P}<0.05$ vs. Ad.V + LPS, normal + LPS, and Ad.rPALM3 + LPS groups; Fig. 3). However, these changes in the levels of cytokines were not observed in the Ad.V-transfected group after LPS challenge ( $\mathrm{P}>0.05$ vs. normal + LPS group; Fig. 3 ).

Effect of PALM3 on the activity of $N F-\kappa B$ in LPS-stimulated rat $A M s . \mathrm{NF}-\kappa \mathrm{B}$ is a critical transcription factor required for the maximal expression of many cytokines (22). As presented in Fig. 4, adenovirus vectors, Ad.V, Ad.rPALM3 and Ad.shRNA, had no impact on NF- $\kappa$ B activity before LPS stimulation ( $\mathrm{P}>0.05$ vs. normal group) (Fig. 4A). All LPS-stimulated rat AMs exhibited a marked increase in NF- $\kappa$ B-DNA-binding in comparison with cells not treated with LPS (Fig. 4). Additionally, Ad.rPALM3 pretreatment further increased the LPS-induced NF- $\kappa \mathrm{B}$ activation $(\mathrm{P}<0.05$ vs. Ad.V + LPS, normal + LPS and Ad.shRNA + LPS groups; Fig. 4). However, Ad.shRNA pretreatment significantly attenuated the LPS-induced increase in NF- $\kappa \mathrm{B}$ activation compared with the Ad.V + LPS and normal + LPS groups ( $\mathrm{P}<0.05$ vs. Ad.V + LPS, normal + LPS and Ad.rPALM3 + LPS groups).

Effect of PALM3 on the IRF3 activity in LPS-stimulated rat $A M s$. IRF3 is also an important transcription factor in cells and serves a role in the post-transcriptional regulation of the proinflammatory cytokines TNF- $\alpha$ and IL-1 $\beta$ (23). In addition, the authors examined the phospho-IRF-3 protein level in nuclear extracts by using western blot analysis to detect the influence of PALM3 on LPS-induced IRF-3 activation. The results demonstrated that the phospho-IRF-3 protein levels in all LPS-stimulated rat AMs were significantly higher than those in normal rat AMs ( $\mathrm{P}<0.05$ vs. normal group; Fig. 5). Additionally, Ad.rPALM3 pretreatment further increased the phospho-IRF-3 protein level in rat AMs after LPS stimulation $(\mathrm{P}<0.05$ vs. Ad.V + LPS, normal + LPS, and Ad.shRNA + LPS groups; Fig. 5). However, Ad.shRNA pretreatment significantly attenuated the phospho-IRF-3 protein level in rat AMs after LPS stimulation, compared with the Ad.V + LPS and normal + LPS groups ( $\mathrm{P}<0.05$ vs. Ad.V + LPS, normal + LPS and Ad.rPALM3 + LPS groups). Pretreatment with Ad.V had 

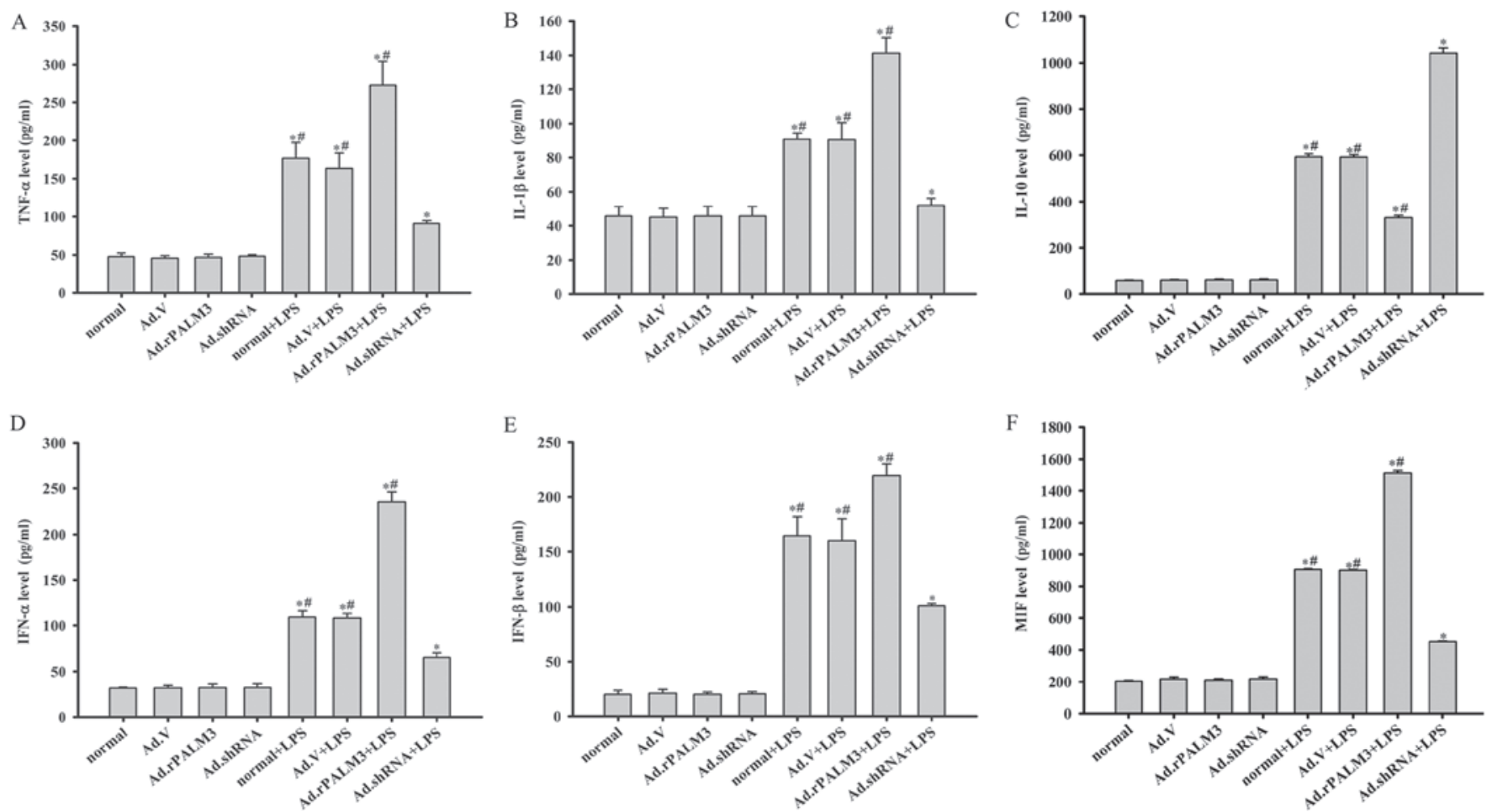

Figure 3. Effect of PALM3 on cytokine production in LPS-stimulated rat AMs. Cells from each group of rat AMs were treated with LPS $(0.5 \mu \mathrm{g} / \mathrm{ml})$ or left untreated. The secretion levels of (A) TNF- $\alpha$, (B) IL-1 $\beta$, (C) IL-10, (D) IFN- $\alpha$, (E) IFN- $\beta$ and (F) MIF were measured by using appropriate ELISA kits. Data are expressed as the mean \pm standard error of the mean of three independent experiments. ${ }^{\text {P }}<0.05$ vs. normal group, Ad.V group, Ad.rPALM3 and Ad.shRNA groups; "P<0.05 vs. Ad.shRNA + LPS group. LPS, lipopolysaccharides; AMs, alveolar macrophages; LPS, lipopolysaccharides; shRNA, short hairpin RNA; Ad, adenovirus; normal, untransfected cells; Ad.shRNA, Ad.shRNA-transfected cells; Ad.V, Ad.V-transfected cells; Ad.rPALM3, Ad.rPALM3transfected cells; normal + LPS, LPS-stimulated untransfected cells; Ad.V + LPS, LPS-stimulated Ad.V-transfected cells; Ad.rPALM3 + LPS, LPS-stimulated Ad.rPALM3-transfected cells; Ad.shRNA + LPS, LPS-stimulated Ad.shRNA-transfected cells; TNF- $\alpha$, tumor necrosis factor- $\alpha$; IL, interleukin; IFN, interferon; MIF, macrophage migration inhibitory factor.

no impact on the LPS-induced increase in phospho-IRF-3 protein level ( $\mathrm{P}>0.05$ vs. normal group; Fig. 5).

Co-localization of TLR4, MyD88, IRAK-1, TRAF-6, and TICAM-2 with PALM3. The cellular localizations of TLR4, MyD88, IRAK-1, TRAF-6, TICAM-2 and PALM3 in NR8383 cells were observed by performing confocal microscopy. Cells were incubated at $4^{\circ} \mathrm{C}$ with anti-TLR4, anti-MyD88, anti-IRAK-1, anti-TRAF-6, anti-TICAM-2 and anti-PALM3 antibodies. Corresponding fluorescence-conjugated secondary antibodies were used to visualize the localization of TLR4, MyD88, IRAK-1, TRAF-6, TICAM-2 and PALM3. The resulting fluorescence signals revealed that, before LPS stimulation, both TLR4 and PALM3 were primarily located on the cell membrane (Fig. 6A1 and A2). When the TLR4-FITC and PALM3-Cy3 localization patterns were superimposed, co-localization of these signals was observed (Fig. 6A3). These results indicated that TLR4 and PALM3 co-localized on the plasma membranes of rat AMs before LPS stimulation. Following LPS stimulation, some TLR4-specific and PALM3specific signals were still observed in the plasma membrane, but they were additionally observed co-localizing in the cell cytoplasm (Fig. 6B1-3). This result suggested that, like the LPS-induced internalization of TLR4, PALM3 also translocated into the cytoplasm in response to LPS stimulation.

The immunofluorescence results also demonstrated that MyD88, IRAK-1, TRAF-6 and TICAM-2 localized in both

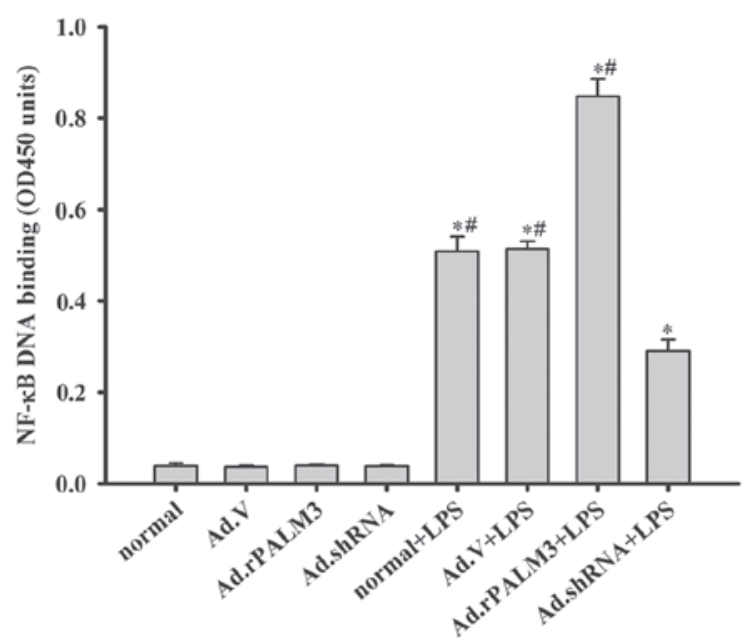

Figure 4. Effect of PALM3 on the NF- $\kappa \mathrm{B}$ activity in LPS-stimulated rat AMs. NF- $\kappa \mathrm{B}$ activity was assessed by performing ELISAs on isolated nuclear extracts of untransfected (normal) or adenovirus-transfected rat AMs that had been stimulated with LPS or left untreated. Data are expressed as the mean \pm standard error of the mean of three independent experiments. ${ }^{*} \mathrm{P}<0.05$ vs. normal group, Ad.V group, Ad.rPALM3, and Ad.shRNA groups; ${ }^{\text {P }}<0.05$ vs. Ad.shRNA + LPS group. AMs, alveolar macrophages; NF- $\mathrm{B}$, nuclear factor- $\kappa \mathrm{B}$; OD, optical density; LPS, lipopolysaccharides; shRNA, short hairpin RNA; Ad, adenovirus; normal, untransfected cells; Ad.shRNA, Ad.shRNA-transfected cells; Ad.V, Ad.V-transfected cells; Ad.rPALM3, Ad.rPALM3-transfected cells; normal + LPS, LPS-stimulated untransfected cells; Ad.V + LPS, LPS-stimulated Ad.V-transfected cells; Ad.rPALM3 + LPS, LPS-stimulated Ad.rPALM3-transfected cells; Ad.shRNA + LPS, LPS-stimulated Ad.shRNA-transfected cells. 


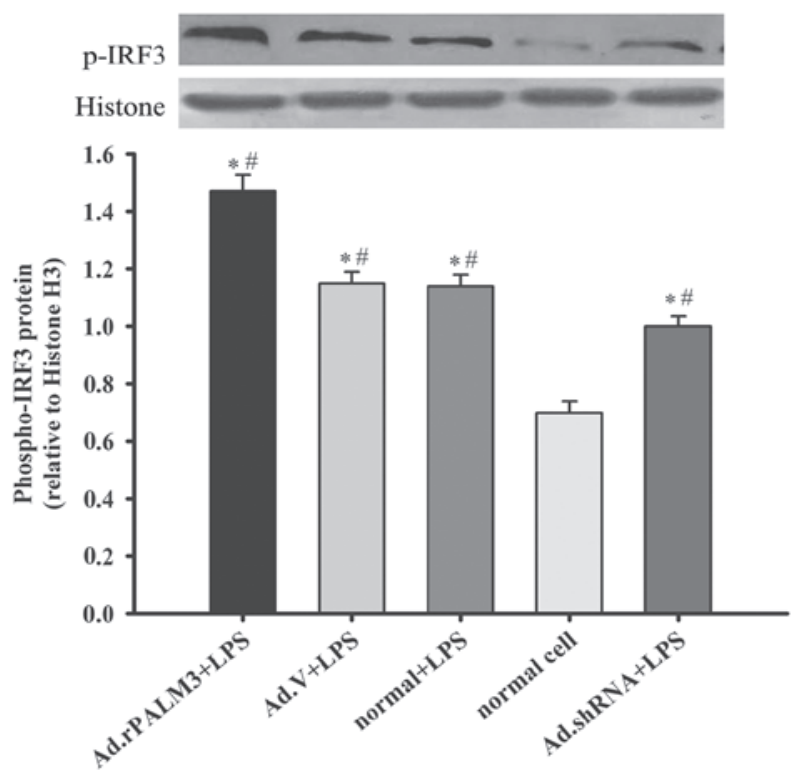

Figure 5. Effect of PALM3 on the IRF-3 activity in LPS-stimulated rat AMs The phosphor-IRF-3 protein levels in nuclear extracts of LPS-stimulated untransfected (normal) or adenovirus-transfected rat AMs were determined by western blotting. The upper panel shows a representative blot, and the lower panel represents densitometry analyses of the bands. Data are expressed as the mean \pm standard error of the mean of three independent experiments. ${ }^{*} \mathrm{P}<0.05$ vs. normal group; ${ }^{\#} \mathrm{P}<0.05$ vs. Ad.shRNA + LPS group. AMs, alveolar macrophages; LPS, lipopolysaccharides; shRNA, short hairpin RNA Ad, adenovirus; normal, untransfected cells; normal + LPS, LPS-stimulated untransfected cells; Ad.V + LPS, LPS-stimulated Ad.V-transfected cells; Ad.rPALM3 + LPS, LPS-stimulated Ad.rPALM3-transfected cells; Ad.shRNA + LPS, LPS-stimulated Ad.shRNA-transfected cells.

the cell cytoplasm and plasma membrane after LPS stimulation (Fig. 6C1-F1). Moreover, as presented in Fig. 6C2-F2, PALM3-specific signals similarly localized not only on the plasma membrane but also in the cell cytoplasm in the presence of LPS. When the MyD88-specific, IRAK-1-specific, TRAF-6-specific or TICAM-2-specific and PALM3-specific signals were superimposed, it was observed that PALM3 co-localized with MyD88, IRAK-1, TRAF-6, and TICAM-2 (Fig. 6C3-F3). Positive staining signals were not observed in the negative controls, which lacked the addition of primary antibody (Fig. 6A4-F4).

PALM3 interacted with the adaptors of LPS-TLR4 signaling. As TLR4, MyD88, IRAK-1, TRAF-6 and Toll-IL-1 receptor containing adapter molecule (TIACM)-2 are believed to be the key adaptor proteins in LPS-TLR4 signaling, the authors used co-immunoprecipitation assays to examine if PALM3 can interact with these adaptors of LPS-TLR4 signaling. Following LPS stimulation, PALM3 protein was detected by western blot analysis after co-precipitation with anti-TLR4, anti-MyD88, anti-IRAK-1, anti-TRAF-6 and anti-TICAM-2 antibodies (Fig. 7). However, when the same assays were conducted on non-LPS-stimulated cells, PALM3 protein was only detected after co-precipitation with an anti-TLR4 antibody (Fig. 7). Notably, PALM3 protein was not detected after co-precipitation with normal nonspecific IgG (Fig. 7). These results suggested that PALM3 interacts with TLR4 under normal conditions, but it also interacts with MyD88, IRAK-1, TRAF-6 and TICAM-2 in a ligand-dependent manner.

\section{Discussion}

In the present study, it was demonstrated that the PALM3 expression level was upregulated by the administration of LPS, and modulating the expression level of PALM3 could affect both the production of cytokines (TNF- $\alpha$, IL-1 $\beta$, IL-10, IFN- $\alpha$, IFN $-\beta$ and MIF) and the activation of NF- $\kappa$ B and IRF-3 in rat AMs after LPS stimulation. Additionally, PALM3 was found to interact with TLR4, IRAK-1, TRAF-6 and TIACM-2 during LPS-TLR4 signaling in rat AMs. To the best of the authors' knowledge, the current study is the first to demonstrate that PALM3 contributes to the LPS-induced inflammatory response and is involved in LPS-TLR4 signaling in AMs.

ALI/ARDS is a lung inflammation disorder with a high mortality rate (2), and its typical pathological characteristic is an excessive lung inflammation response (24). LPS-TLR4 signaling is one of the most important inflammatory response signal pathways (25). As AMs are the major effector cells that participate in the initiation and development of ALI $(4,5,26)$, modulating the excessive inflammatory response to pathogenassociated molecular patterns in AMs during systemic inflammatory response syndrome is a therapeutic target for treating ALI/ARDS. PALM3 is a novel interactive partner of single immunoglobulin IL-1 receptor-related molecule (SIGIRR) and may function as an adaptor in LPS-TLR4 signaling (19). Downregulation of PALM3 has been reported to benefit mice with LPS-induced ALI by means of suppressing the inflammatory response, ameliorating histological tissue injury, and decreasing the permeability of the alveolar capillary barrier (20). Additionally, the preliminary data also showed that the downregulation of PALM3 protected rats from LPS-induced ALI, and its mechanisms were partially associated with the modulation of cytokine secretion and inhibition of NF- $\mathrm{B}$ and IRF3 activation. In the present study, the authors indicated that the downregulation of PALM3 significantly suppressed the inflammatory response to LPS in AMs. Therefore, inhibiting the LPS-induced inflammatory response in AMs may be part of the mechanism for the protective effect of PALM3-knockdown on ALI.

At present, little is understood regarding the biological function of the PALM3. Previous studies have demonstrated that PALM3 may act as an adaptor to link intrinsic membrane proteins to each other, to the cytoskeleton, or to motor proteins $(14,19)$. Previous work of the authors revealed that LPS upregulated the PALM3 expression in alveolar epithelial cells (19). Furthermore, the present results show that PALM3 expression was detected and also upregulated in a time-dependent manner in rat AMs following LPS stimulation, which is similar to previous results in alveolar epithelial cells (19). The PALM3 expression pattern is similar to the expression patterns of TLR4, TIR domain-containing adaptor inducing IFN- $\beta$ (TRIF) and MyD88 $(27,28)$, and the upregulation of PALM3 may be related to its functional involvement in LPS-TLR4 signal transduction, similar to the adaptors in TLR signaling (19). To elucidate the role of PALM3 in LPS-TLR4 signaling in AMs, the authors used a recombinant adenovirus expressing shRNA for PALM3 (Ad.shRNA) and a recombinant adenovirus carrying the rat $P A L M 3$ gene (Ad.rPALM3) to modulate the expression of PALM3 in rat AMs. The results of western blotting and RT-qPCR analyses 
Non-LPS
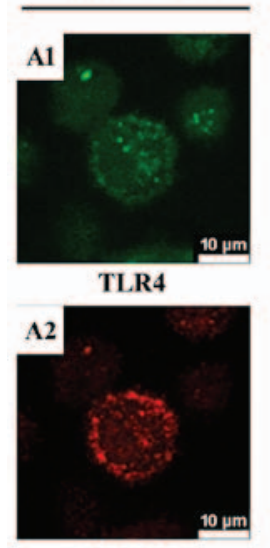

PALM3
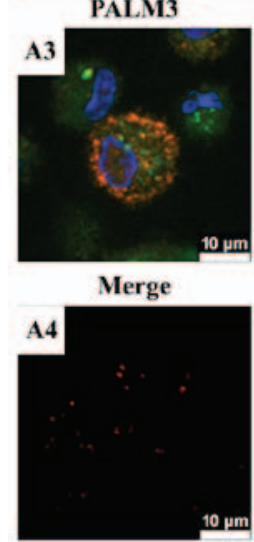

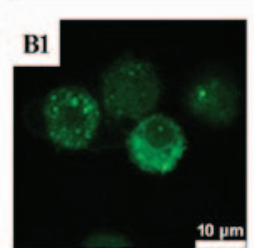

TLR4

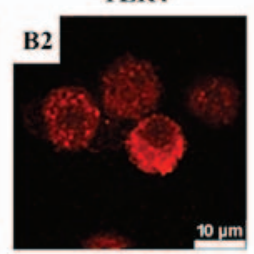

PALM3
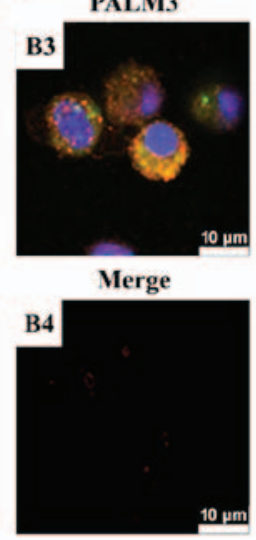
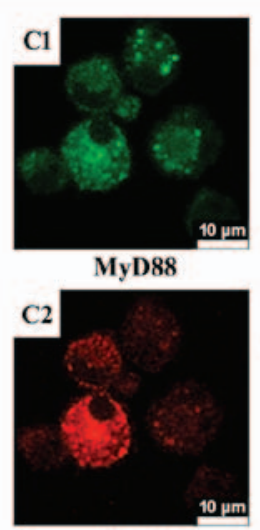

PALM3
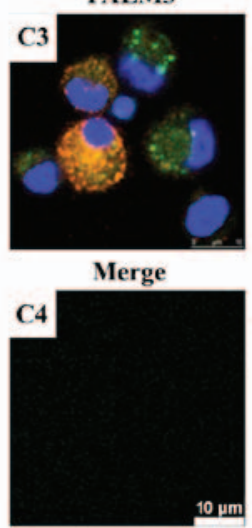

LPS
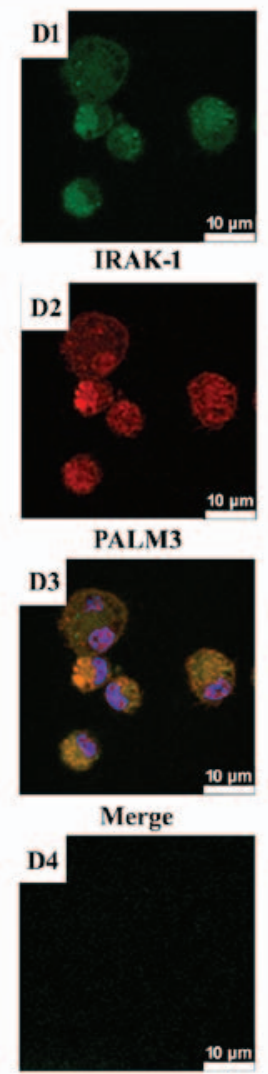
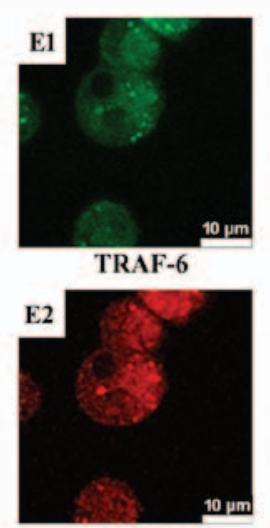

PALM3
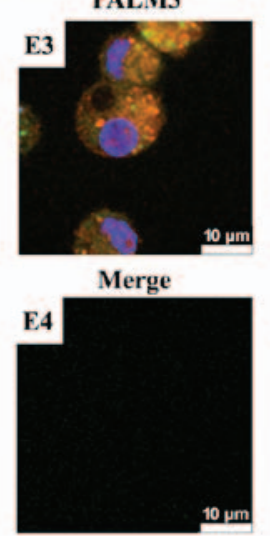

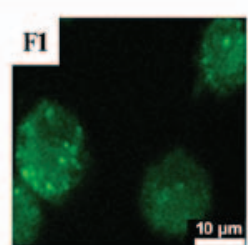

TICAM-2

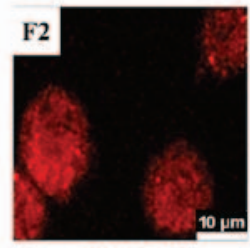

PALM3
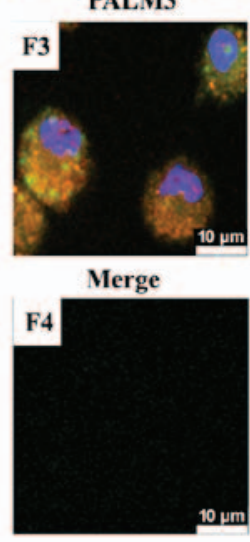

Figure 6. Localization of PALM3 and LPS-TLR4 signaling adaptor proteins in rat AMs. Rat AMs were incubated with anti-PALM3 antibody along with anti-TLR4, anti-MyD88, anti-IRAK-1, anti-TRAF-6, or anti-TICAM-2 antibodies, followed by incubation with corresponding fluorescein isothiocyanateconjugated or Cy3-conjugated secondary antibodies. Nuclei were counterstained with DAPI. The TLR4-, MyD88-, IRAK-1-, TRAF-6- and TICAM-2-specific signals resulting from the immunofluorescence staining are shown in green (A1, B1, C1, D1, E1, F1), and the PALM3-specific signals are shown in red (A2, B2, C2, D2, E2, F2). The resulting merged images highlight co-localizations (shown in yellow) of the signals for TLR4, MyD88, IRAK-1, TRAF-6, or TICAM-2 and PALM3 (A3, B3, C3, D3, E3, F3). Negative controls, which were not treated with primary antibodies, are shown in A4, B4, C4, D4, E4, F4. LPS, lipopolysaccharides; TLR4, Toll-like receptor 4; AMs, alveolar macrophages; MyD88, myeloid differentiation factor 88; IRAK, interleukin 1 receptor associated kinase; TRAF, tumor necrosis factor receptor associated factor; TICAM2, Toll-like receptor adaptor molecule 2.

demonstrate that using these recombinant adenoviral vectors successfully modulated the PALM3 gene transcript in AMs.

Previous studies have demonstrated that the downregulation of PALM3 inhibits the LPS-induced release of inflammatory cytokines in alveolar epithelial cells and had a protective effect against LPS-induced ALI in mice $(19,20)$. In the current study, we identified a similar result in rat AMs. That is, the downregulation of PALM3 by transfection with Ad.shRNA suppressed the release of proinflammatory cytokines (TNF- $\alpha$, IL- $1 \beta$, IFN- $\alpha$, IFN- $\beta$ and MIF), but the upregulation of PALM3 by transfection with Ad.rPALM3 promoted the production of proinflammatory cytokines (TNF- $\alpha$, IL-1 $\beta$, IFN- $\alpha$, IFN- $\beta$ and MIF) in LPS-stimulated AMs. The increase in the production of proinflammatory cytokines in Ad.rPALM3-pretreated cells may be due to the promotion of LPS-TLR4 signal transduction by the enhanced expression of PALM3. Correspondingly, the downregulation of PALM3 may decrease the promotion effect of PALM3 on proinflammatory cytokine secretion. In contrast, modulating the PALM3 expression level had the opposite effect on the secretion of the anti-inflammatory cytokine IL-10. Moreover, the authors examined the effect of PALM3 on the activation of $N F-\kappa B$ and IRF-3. The results demonstrated that the activity levels of transcription factor IRF-3 and NF- $\kappa$ B were significantly strengthened in Ad.rPALM3-pretreated cells following LPS stimulation, compared with the Ad.V-pretreated cells. Correspondingly, the downregulation of PALM3 by pretreatment with Ad.shRNA suppressed the NF- $\kappa$ B-DNA-binding activity and decreased the phospho-IRF3 protein levels in the nucleus of rat AMs. These changes in the IRF-3 and NF- $\kappa$ B activity support the idea that enhanced PALM3 expression may promote the transduction of LPS-TLR4 signaling while interfering with PALM3 expression might impede this signal transduction. Determining the detailed molecular mechanisms of this process will require further investigations.

Protein-protein interaction is an important molecular mechanism in signal transduction (29). LPS can induce the dimerization of TLR4, which results in conformational changes of the TLR4 homodimer that induce the recruitment of adaptor proteins containing Toll/interleukin-1 receptorlike (TIR) domains. In the MyD88-dependent pathway, the TLR4 TIR domains recruit TIR domain-containing adaptor proteins MyD88-adaptor-like (MAL) and MyD88, and in the MyD88-independent pathway, these domains recruit TIR domain-containing adaptor inducing IFN- $\beta$ (TRIF) and TICAM-2 (11). The MyD88-dependent pathway involves the 

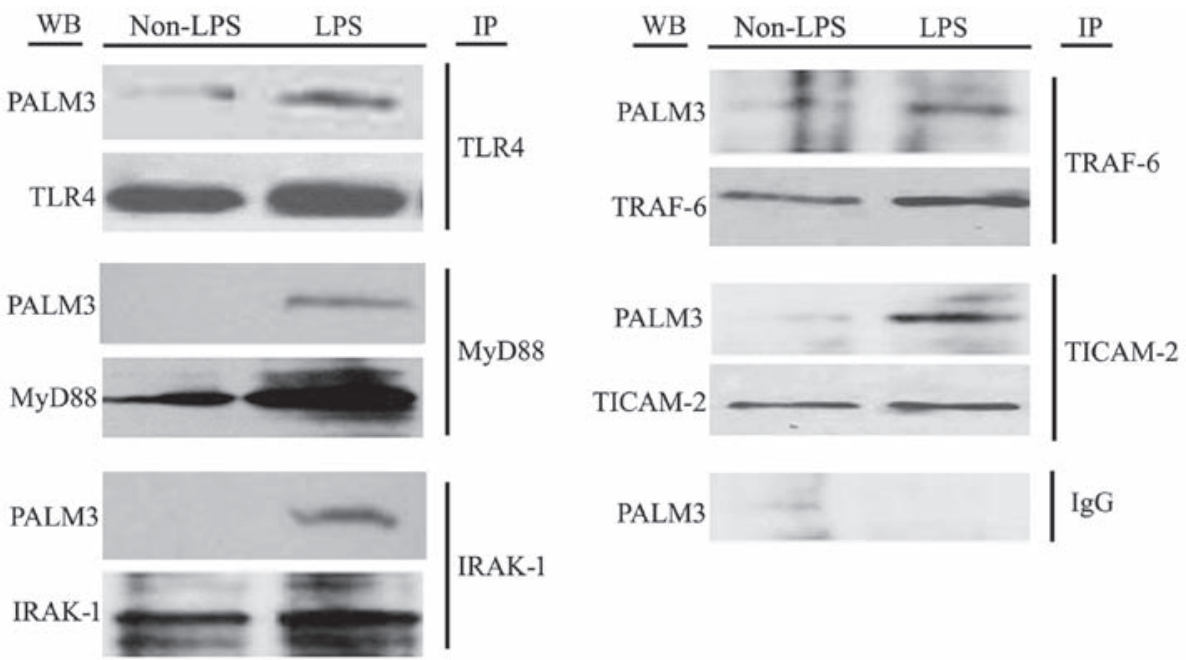

Figure 7. Co-immunoprecipitation assays of TLR4, MyD88, IRAK-1, TRAF-6 and TICAM-2 with PALM3 in LPS-stimulated rat AMs. IP, immunoprecipitation: the total cell lysates were immunoprecipitated with anti-TLR4, anti-MyD88, anti-IRAK-1, anti-TRAF-6, anti-TICAM-2 or normal IgG antibodies; WB, western blot analysis: the immunocomplexes were examined by western blot analysis using the indicated antibodies; TLR4, Toll-like receptor 4; AMs, alveolar macrophages; MyD88, myeloid differentiation factor 88; IRAK, interleukin 1 receptor associated kinase; TRAF, tumor necrosis factor receptor associated factor; TICAM2, Toll-like receptor adaptor molecule 2; LPS, lipopolysaccharide; AMs, alveolar macrophages; IgG, immunoglobulin G.

recruitment and activation of IRAKs and TRAF-6, and the activation of this pathway induces the activation and translocation in the nucleus of transcription factors, such as $\mathrm{NF}-\kappa \mathrm{B}$ and activator protein-1 (AP-1) (11). The MyD88-independent pathway involves TICAM-1 and TICAM-2 adaptor proteins, and the activation of this signaling pathway leads to the activation and translocation in the nucleus of the transcription factor IRF3 (30). Furthermore, the MyD88-dependent pathway induces the production of proinflammatory cytokines, while the MyD88-independent pathway induces the production of type I interferons (11).

In the previous study, the authors found that PALM3 can interact with SIGIRR, a negative regulator of TLR signaling (19). Based on what is currently known about SIGIRR, the authors presumed that, as an interaction partner of SIGIRR, PALM3 may be involved in TIR signaling. Additionally, because PALM3 can function as an adaptor that links intrinsic membrane proteins to each other (14), it was also suspected that PALM3 may function as an adaptor in TLR signaling. Herein, the results of co-immunoprecipitation assays showed that PALM3 interacted with TLR4, IRAK-1, TRAF-6 and TIACM-2 in rat AMs following LPS stimulation. Moreover, the interaction between PALM3 and TLR4 was independent of LPS stimulation. Meanwhile, the results of immunofluorescence staining revealed that PALM3 and TLR4 proteins co-localize in rat AMs whether or not the cells are stimulated by LPS, demonstrating that PALM3 is located in the cell membrane under normal conditions and can internalize into the cell cytoplasm with the translocation of TLR4 after LPS stimulation. Together, these results suggested that the interaction between PALM3 and TLR4 exists in the natural state, independent of LPS stimulation.

Past work has indicated that the adaptor proteins MyD88, IRAK-1, TRAF-6 and TIACM-2 are located in the cell cytoplasm in the natural state $(11,31)$, and these adaptors can be recruited to the cell membrane through homophilic interactions between TIR domains in the cytoplasmic tail of TLR4 and those present on the adaptors after LPS stimulation (11). Our findings are similar to the results of these previous studies (11,31). The adaptor proteins MyD88, IRAK-1, TRAF-6 and TIACM-2 were localized in the cell cytoplasm and on the plasma membrane in the presence of LPS. This supports the spatial possibility that an interaction between PALM3 and the adaptor proteins, i.e. MyD88, IRAK-1, TRAF-6 and TIACM-2, exists in the presence of LPS. All of these data support the idea that PALM3 can interact with TLR4, MyD88, IRAK-1, TRAF-6 and TIACM-2 in rat AMs. Notably, MyD88, IRAK-1 and TRAF-6 are adaptor proteins of the MyD88-dependent pathway, but TIACM-2 is an adaptor protein of the MyD88-independent pathway $(11,30)$. Therefore, the current results suggested that PALM3 may function as an adaptor that participates in both the MyD88-dependent and MyD88-independent pathways. However, whether or not PALM3 serves the same role in LPS-TLR4 signaling and possess similar regulation effects in an ALI animal model warrants further investigation. Additionally, the authors used a rat NR8383 macrophage cell line to investigate the role of PALM3 in LPS-TLR4 signaling in the present study. Further experiments in primary AMs isolated from an ALI animal model will be needed to confirm these findings on the regulation effect of PALM3 on LPS-TLR4 signaling.

TLR4 belongs to a family of pattern recognition receptors and has been recognized as the sensing receptor for LPS (11). In the present study, the results showed that PALM3 co-localized and interacted with TLR4 protein in rat AMs in an LPS-independent manner. In contrast, the interaction between PALM3 and the adaptor proteins, MyD88, IRAK-1, TRAF-6 and TIACM-2, depended on the presence of LPS. In addition, previous studies have demonstrated that PALM3 interacts with SIGIRR (a negative regulator of TLR signaling) (19) and functions as an adaptor that links intrinsic membrane proteins to each other (14). Therefore, it was proposed that PALM3 may act as an 'adaptor' or a 'bridge' between TLR4 and the proteins MyD88, IRAK-1, TRAF-6 
and TIACM-2 in the LPS-TLR4 signal transduction pathway, which may enhance the interaction between TLR4 and these adaptor proteins. Subsequently, this interaction enhancement may further lead to inflammation. The results do not reveal whether or not the interaction between PALM3 and the adaptor proteins, MyD88, IRAK-1, TRAF-6 and TIACM-2, depends on TLR4. This question remains to be confirmed by experiments using small interfering RNA or an inhibitor to downregulate TLR4. Although it is believed that this issue is an important subject worth studying in LPS-TLR4 signaling, it was not the focus of the present study. Moreover, the mechanisms relevant to the interactions between the adaptors and LPS-induced inflammatory signaling are very complex, beyond simply the effect of TLR4. Therefore, a separate, but more extensive, study of this topic is planned in the future. In conclusion, the authors have demonstrated that PALM3 can interact with TLR4, MyD88, IRAK-1, TRAF-6 and TIACM-2 after LPS stimulation and that upregulation of PALM3 aggravates the LPS-induced inflammation, while downregulation of PALM3 suppresses the LPS-induced inflammation in rat AMs. Therefore, the authors hypothesized that PALM3 may function as an adaptor to participate in the transduction of LPS-TLR4 signaling and contribute to LPS-induced inflammatory responses in AMs. Modulating PALM3 expression may be a potential novel target for treating macrophageassociated inflammatory diseases. Future research will focus on the issues emerging from this study and on the detailed mechanisms by which PALM3 participates in LPS-TLR4 signal transduction.

\section{Acknowledgements}

The present study was supported by a grant from the National Natural Science Foundation of China (grant no. 81300050) and the Innovative Cultivation Foundation of Navy General Hospital of PLA (grant no. CXPY201417).

\section{References}

1. Wang Q, Wang J, Hu M, Yang Y, Guo L, Xu J, Lei C, Jiao Y and $\mathrm{Xu}$ J: Uncoupling protein 2 increases susceptibility to lipopolysaccharide-induced acute lung injury in mice. Mediators Inflamm 2016: 9154230, 2016.

2. Wang Q, Zheng X, Cheng Y, Zhang YL, Wen HX, Tao Z, Li H, Hao Y, Gao Y, Yang LM, et al: Resolvin D1 stimulates alveolar fluid clearance through alveolar epithelial sodium channel, Na,K-ATPase via ALX/cAMP/PI3K pathway in lipopolysaccharide-induced acute lung injury. J Immunol 192 3765-3777, 2014.

3. Zhu T, Wang DX, Zhang W, Liao XQ, Guan X, Bo H, Sun JY, Huang NW, He J, Zhang YK, et al: Andrographolide protects against LPS-induced acute lung injury by inactivation of NF- $\kappa \mathrm{B}$. PLoS One 8: e56407, 2013.

4. Han J, Li C, Liu H, Fen D, Hu W, Liu Y, Guan C and Luo ZQ: Inhibition of lipopolysaccharide-mediated rat alveolar macrophage activation in vitro by antiflammin-1. Cell Biol Int 32: 1108-1115, 2008.

5. Wu DD, Pan PH, Liu B, Su XL, Zhang LM, Tan HY, Cao Z, Zhou ZR, Li HT, Li HS, et al: Inhibition of alveolar macrophage pyroptosis reduces lipopolysaccharide-induced acute lung injury in mice. Chin Med J (Engl) 128: 2638-2645, 2015.

6. Johnston LK, Rims CR, Gill SE, McGuire JK and Manicone AM: Pulmonary macrophage subpopulations in the induction and resolution of acute lung injury. Am J Respir Cell Mol Biol 47: 417-426, 2012.

7. Aggarwal NR, King LS and D'Alessio FR: Diverse macrophage populations mediate acute lung inflammation and resolution. Am J Physiol Lung Cell Mol Physiol 306: L709-L725, 2014.
8. Dagvadorj J, Shimada K, Chen S, Jones HD, Tumurkhuu G, Zhang W, Wawrowsky KA, Crother TR and Arditi M: Lipopolysaccharide induces alveolar macrophage necrosis via $\mathrm{CD} 14$ and the $\mathrm{P} 2 \mathrm{X} 7$ receptor leading to interleukin-1 $\alpha$ release. Immunity 42: 640-653, 2015.

9. Takashima K, Matsushima M, Hashimoto K, Nose H, Sato M, Hashimoto N, Hasegawa Y and Kawabe T: Protective effects of intratracheally administered quercetin on lipopolysaccharideinduced acute lung injury. Respir Res 15: 150, 2014.

10. Xi F, Liu Y, Wang X, Kong W and Zhao F: LYATK1 potently inhibits LPS-mediated pro-inflammatory response. Biochem Biophys Res Commun 470: 1-8, 2016.

11. Molteni M, Gemma S and Rossetti C: The role of Toll-like receptor 4 in infectious and noninfectious inflammation. Mediators Inflamm 2016: 6978936, 2016.

12. Bordon Y: Inflammation: TNF snuffs out steroids. Nat Rev Immunol 14: 214, 2014.

13. Cornish JA, Kloc M, Decker GL, Reddy BA and Etkin LD: Xlcaax-1 is localized to the basolateral membrane of kidney tubule and other polarized epithelia during Xenopus development. Dev Biol 150: 108-120, 1992.

14. Hu B, Petrasch-Parwez E, Laue MM and Kilimann MW: Molecular characterization and immunohistochemical localization of palmdelphin, a cytosolic isoform of the paralemmin protein family implicated in membrane dynamics. Eur J Cell Biol 84: 853-866, 2005.

15. Kutzleb C, Sanders G, Yamamoto R, Wang X, Lichte B, Petrasch-Parwez E and Kilimann MW: Paralemmin, a prenylpalmitoyl-anchored phosphoprotein abundant in neurons and implicated in plasma membrane dynamics and cell process formation. J Cell Biol 143: 795-813, 1998.

16. Albrecht I, Bieri R, Leu A, Granacher P, Hagmann J, Kilimann MW and Christofori G: Paralemmin-1 is expressed in lymphatic endothelial cells and modulates cell migration, cell maturation and tumor lymphangiogenesis. Angiogenesis 16: 795-807, 2013

17. Turk CM, Fagan-Solis KD, Williams KE, Gozgit JM, SmithSchneider S, Marconi SA, Otis CN, Crisi GM, Anderton DL, Kilimann MW, et al: Paralemmin-1 is over-expressed in estrogen-receptor positive breast cancers. Cancer Cell Int 12: 17, 2012.

18. Kutzleb C, Petrasch-Parwez E and Kilimann MW: Cellular and subcellular localization of paralemmin-1, a protein involved in cell shape control, in the rat brain, adrenal gland and kidney. Histochem Cell Biol 127: 13-30, 2007.

19. Chen X, Wu X, Zhao Y, Wang G, Feng J, Li Q and Qian G: A novel binding protein of single immunoglobulin IL-1 receptorrelated molecule: Paralemmin-3. Biochem Biophys Res Commun 404: 1029-1033, 2011

20. Li S, Guo L, Zhao Y, Qian P, Lv X, Qian L, Wang Q, Qian G, Yao $\mathrm{W}$ and $\mathrm{Wu} X$ : Silencing of paralemmin-3 protects mice from lipopolysaccharide-induced acute lung injury. Peptides 76: 65-72, 2016.

21. Livak KJ and Schmittgen TD: Analysis of relative gene expression data using real-time quantitative PCR and the 2(-Delta Delta C(T)) Method. Methods 25: 402-408, 2001.

22. Rao R, Nagarkatti P and Nagarkatti M: Role of miRNA in the regulation of inflammatory genes in staphylococcal enterotoxin B-induced acute inflammatory lung injury and mortality. Toxicol Sci 144: 284-297, 2015.

23. Liu LM, Tu WJ, Zhu T, Wang XT, Tan ZL, Zhong H, Gao DY and Liang DY: IRF3 is an important molecule in the UII/UT system and mediates immune inflammatory injury in acute liver failure. Oncotarget 7: 49027-49041, 2016.

24. Qi T, Xu F, Yan X, Li S and Li H: Sulforaphane exerts antiinflammatory effects against lipopolysaccharide-induced acute lung injury in mice through the Nrf2/ARE pathway. Int J Mol Med 37: 182-188, 2016.

25. Sugiyama K, Muroi M, Kinoshita M, Hamada O, Minai Y, Sugita-Konishi Y, Kamata Y and Tanamoto K: NF- $\kappa B$ activation via MyD88-dependent Toll-like receptor signaling is inhibited by trichothecene mycotoxin deoxynivalenol. J Toxicol Sci 41: 273-279, 2016.

26. Fernandez-Bustamante A, Agazio A, Wilson P, Elkins N, Domaleski L, He Q, Baer KA, Moss AF, Wischmeyer PE and Repine JE: Brief glutamine pretreatment increases alveolar macrophage CD163/heme oxygenase-1/p38-MAPK dephosphorylation pathway and decreases capillary damage but not neutrophil recruitment in IL-1/LPS-insufflated rats. PLoS One 10: e0130764, 2015. 
27. Shan Y, Lin N, Yang X, Tan J, Zhao R, Dong S and Wang S: Sulphoraphane inhibited the expressions of intercellular adhesion molecule-1 and vascular cell adhesion molecule-1 through MyD88-dependent toll-like receptor-4 pathway in cultured endothelial cells. Nutr Metab Cardiovasc Dis 22: 215-222, 2012.

28. Van Linthout S, Spillmann F, Graiani G, Miteva K, Peng J, Van Craeyveld E, Meloni M, Tölle M, Escher F, Subasigüller A, et al: Down-regulation of endothelial TLR4 signalling after apo A-I gene transfer contributes to improved survival in an experimental model of lipopolysaccharide-induced inflammation. J Mol Med (Berl) 89: 151-160, 2011.
29. Safari-Alighiarloo N, Taghizadeh M, Rezaei-Tavirani M, Goliaei B and Peyvandi AA: Protein-protein interaction networks (PPI) and complex diseases. Gastroenterol Hepatol Bed Bench 7: 17-31, 2014

30. Oshiumi H, Sasai M, Shida K, Fujita T, Matsumoto M and Seya T: TIR-containing adapter molecule (TICAM)-2, a bridging adapter recruiting to toll-like receptor 4 TICAM-1 that induces interferon-beta. J Biol Chem 278: 49751-49762, 2003.

31. Wald D, Qin J, Zhao Z, Qian Y, Naramura M, Tian L, Towne J, Sims JE, Stark GR and Li X: SIGIRR, a negative regulator of Toll-like receptor-interleukin 1 receptor signaling. Nat Immunol 4: 920-927, 2003. 